NASA $-C R-$

180882

AIAA-88-0436

\title{
Numerical Study of Chemically Reacting Flows Using an LU Scheme
}

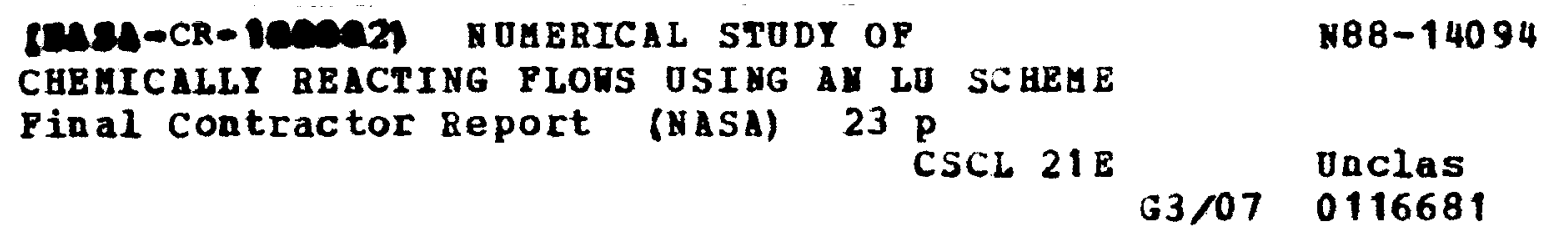

Jian Shun Shuen

Sverdrup Technology, Inc.

Lewis Research Center

Cleveland, Ohio

and

Seokkwan Yoon

MCAT Institute

Ames Research Center

Moffett Field, California

Prepared for

Lewis Research Center

Under Contract NAS3-24105 
ERRATA

NASA Technical Memorandum 180882

NUMERICAL STUDY OF CHEMICALLY REACTING FLOWS

USING AN LU SCHEME

$$
\begin{gathered}
\text { Jian Shun Shuen } \\
\text { and } \\
\text { Seokkwan Yoon } \\
\text { January } 1988
\end{gathered}
$$

Cover and Report Documentation Page (Box 1): NASA Technical Memorandum 180882 should be NASA Contractor Report 180882. 
NUMERICAL STUDY OF CHEMICALLY REACTING FLOWS USING AN LU SCHEME

\author{
Jian Shun Shuen* \\ Sverdrup Technology, Inc. \\ Lewis Research Center \\ Cleveland, Ohio 44135 \\ Seokkwan Yoon* \\ MCAT Institute \\ Ames Research Center \\ Moffett Field, California 94035
}

\title{
SUMMARY
}

A new computational fluid dynamic code has been developed for the study of mixing and chemical reactions in the flow fields of ramjets and scramjets. The code employs an implicit finite volume, lower-upper symmetric successive overrelaxation scheme (LU-SSOR) for solving the complete two-dimensional Navier-Stokes equations and species transport equations in a fully-coupled and very efficient manner. The combustion processes are modeled by an 8-species, 14-step finite-rate chemistry model whereas turbulence is simulated by a Baldwin-Lomax algebraic model. The validity of the code is demonstrated by comparing the numerical calculations with both experimental data and previous calculations of a cold flow helium injection into a straight channel and premixed hydrogen-air reacting flows in a ramped duct. The code is then used to calculate the mixing and chemical reactions of a hydrogen jet transversely injected into a supersonic air stream. Results are presented that describe the flow field, the recirculation regions in front and behind the injector, and the chemical reactions.

\section{INTRODUCTION}

The recent interest in hypersonic vehicles has created a need for efficient numerical methods for the prediction of turbulent mixing and combustion in transonic and supersonic flows. In developing such a predictive method, numerical stability and efficiency are two important aspects that have to be considered. The stability problem arises mainly from the stiffness of the chemical source terms in the species concentration equations. The concern over the numerical efficiency, on the other hand, is mainly caused by the large number of species equations that have to be solved along with the flow equations and the close coupling between the flow and the chemistry. In recent years a number of numerical methods (refs. 1 to 4 ), have been developed for the calculation of steady-state supersonic reacting flows. Most of these methods have treated chemical source terms implicitly to remove the stiffness associated with the species equations. While this approach (treating chemical source terms implicitly) has been successful in circumventing the stiffness limitation, most existing methods have suffered from numerical inefficiency. The

*Member AIAA. 
majority of the reacting flow codes developed to date has adopted explicit integration methods in which the flow and the species equations are solved sequentially and are not directly coupled. Since in most chemically reacting flows the coupling between species and flow variables is strong, explicit methods are generally not robust and very slow in convergence. Most implicit methods, such as the popular Beam and Warming scheme (ref. 5), on the other hand, require the inversion of large block matrices. This can be very costly when a large number of species equations are solved in a coupled manner with the flow equations.

In the present paper a new numerical method is described for the study of mixing and chemical reactions in transonic and supersonic flows. This method employs an implicit finite volume, lower-upper symmetric overrelaxation (LU-SSOR) scheme for solving the complete two-dimensional Navier-Stokes equations and species transport equations in a fully-implicit and fully-coupled manner, with real gas properties. Despite being implicit, the LU scheme requires only scalar diagonal inversions while most other implicit schemes require block matrix inversions. The use of scalar diagonal inversions offers large savings in computer CPU time. The LU scheme has recently been developed for nonreacting flows by the second author (refs. 6 and 7). Extensive tests have shown that this scheme is very robust and efficient for transonic and supersonic nonreacting flows. The objective of the present paper is to demonstrate the capability of the new LU scheme for chemically reacting flows.

Several mixing and/or combustion problems are considered in this study, including a cold flow helium injection, premixed hydrogen and a ir reacting flows in a ramped duct, and a transverse injection of hydrogen into a supersonic airstream with combustion. The chemical reactions are represented by an 8-species, 14-step chemistry model of Westbrook (ref. 8) and Hitch et al. (ref. 9). The governing equations are Reynolds-averaged, and the closure of the equations is provided by the algebraic eddy viscosity model of Baldwin and Lomax (ref. 10).

\section{NOMENCLATURE}

$C_{p_{j}} \quad$ constant pressure specific heat of species $i$

$C_{v_{j}} \quad$ constant volume specific heat of species $i$

$D_{i j}$ binary diffusion coefficient

e total internal energy

$h_{j}$ enthalpy of species $i$

$h_{f_{i}}^{0}$ heat of formulation of species $i$

I identity matrix 


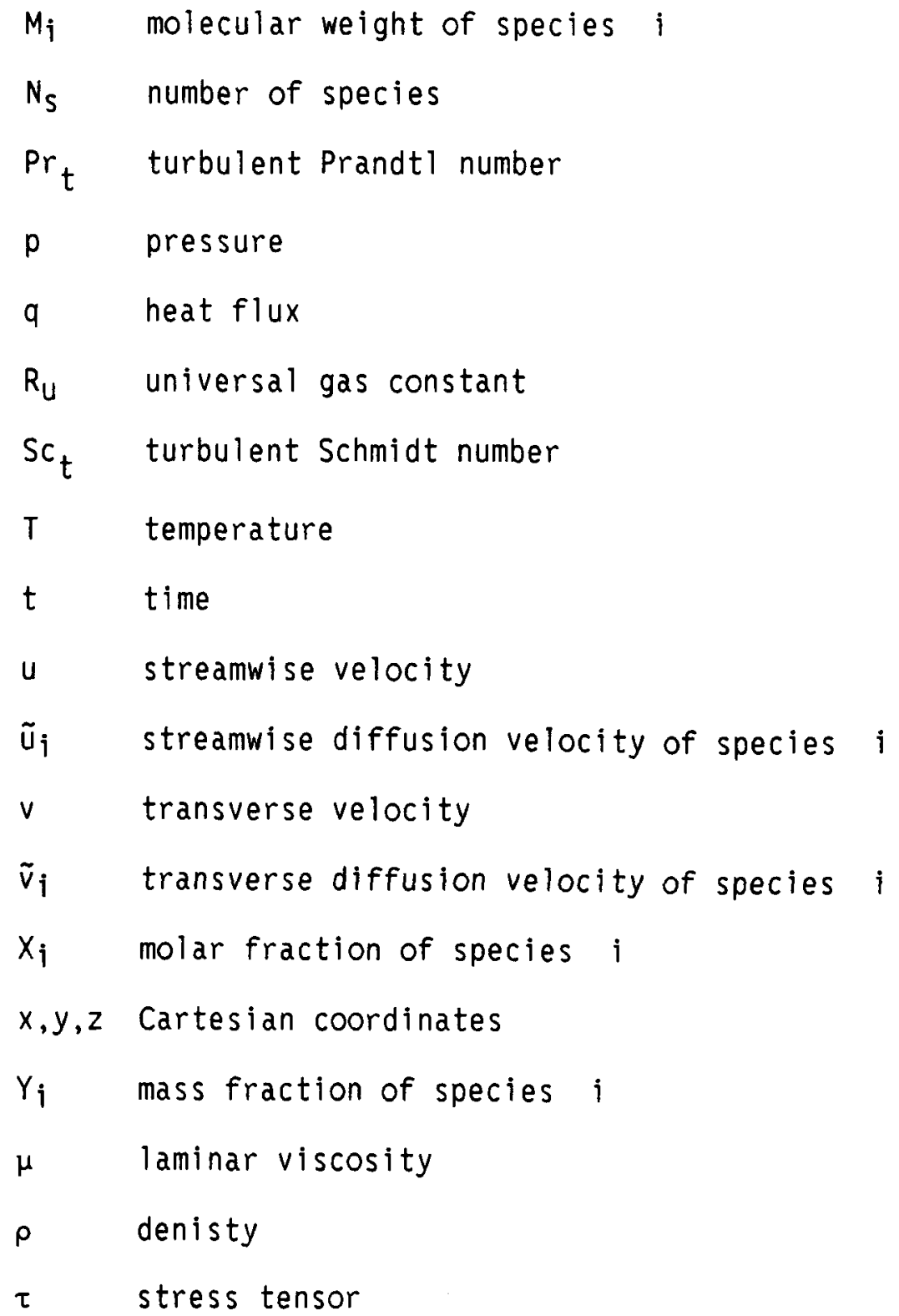

Subscripts:

$i, j \quad x$ and $y$ grid indices

\section{Superscripts:}

n time index

ANALYSIS

Governing equations

The two-dimensional unsteady Navier-Stokes and species transport equations for chemically reacting flows, in Cartesian cordinates, are given by (refs. 11 and 12): 


$$
\frac{\partial Q}{\partial t}+\frac{\partial\left(F-F_{v}\right)}{\partial x}+\frac{\partial\left(G-G_{v}\right)}{\partial y}=S
$$

where

$$
Q=\left[\begin{array}{c}
\rho \\
\rho u \\
\rho v \\
\rho e \\
\rho Y_{i}
\end{array}\right] \quad F=\left[\begin{array}{c}
\rho u \\
\rho u^{2}+p \\
\rho u v \\
u(\rho e+p) \\
\rho u_{i}
\end{array}\right] \quad G=\left[\begin{array}{c}
\rho v \\
\rho u v \\
\rho v^{2}+p \\
v(\rho e+p) \\
\rho v Y_{i}
\end{array}\right]
$$$$
F_{v}=\left[\begin{array}{c}
0 \\
\tau_{x x} \\
\tau_{x y} \\
u \tau_{x x}+v \tau_{x y}-q_{x} \\
-\rho \tilde{u}_{i Y}
\end{array}\right] \quad G_{v}=\left[\begin{array}{c}
0 \\
\tau_{x y} \\
\tau_{y y} \\
u \tau_{x y}+v \tau_{y y}-q_{y} \\
-\rho \tilde{v}_{Y Y}
\end{array}\right]
$$

$$
S=\left[\begin{array}{l}
0 \\
0 \\
0 \\
0 \\
\dot{W}_{i}
\end{array}\right], i=1,2, \ldots, N_{s}-1
$$

and

$$
\begin{aligned}
& \tau_{x x}=2 \mu u_{x}-\frac{2}{3} \mu\left(u_{x}+v_{y}\right) \\
& \tau_{x y}=\mu\left(u_{y}+v_{x}\right) \\
& \tau_{y y}=2 \mu v_{y}-\frac{2}{3} \mu\left(u_{x}+v_{y}\right)
\end{aligned}
$$




$$
\begin{aligned}
& q_{x}=-k \frac{\partial T}{\partial x}+\rho \sum_{i=1}^{N_{s}} h_{i} Y_{i} \tilde{u}_{i} \\
& q_{y}=-k \frac{\partial T}{\partial y}+\rho \sum_{i=1}^{N_{s}} h_{i} Y_{i} \tilde{v}_{i}
\end{aligned}
$$

The diffusion velocities are found by Fick's law

$$
Y_{i} \tilde{u}_{i}=-D_{i m} \frac{\partial}{\partial x} Y_{i} \quad Y_{i} \tilde{v}_{i}=-D_{i m} \frac{\partial}{\partial y} Y_{i}
$$

where $D_{i m}=\left(1-x_{i}\right) \int_{j \neq i}^{\sum}\left(x_{i} / D_{i j}\right)$ is the effective binary diffusivity of species $\mathfrak{i}$ in the gas mixture (ref. 12). The temperature and pressure are calculated iteratively from the following equations

$$
\begin{gathered}
e=\sum_{i=1}^{N_{s}} Y_{i} h_{i}-\frac{p}{\rho}+\frac{1}{2}\left(u^{2}+v^{2}\right) \\
h_{i}=h_{f_{j}}^{0}+\int_{T_{R e f}}^{T} C_{p_{j}} d T \\
p=\rho R_{u} \sum_{i=1}^{N_{s}} \frac{Y_{i}}{M_{i}}
\end{gathered}
$$

Thermodynamics and Transport Models

The specific heat, thermal conductivity, and viscosity for each species are determined by fourth-order polynomials of temperature. The coefficients of these polynomials are supplied by McBride (ref. 13) and are valid up to temperature of $6000 \mathrm{~K}$. The specific heat of the gas mixture is obtained by mass concentration weighting of each species. The thermal conductivity and viscosity of the mixture, however, are calculated using Wilke's mixing rule (ref. 14).

The binary mass diffusivity $D_{i j}$ between species $i$ and $j$ is obtained using the Chapman-Enskog theory in conjunction with the Lennard-Jones intermolecular potential functions (ref. 14). The diffusion of a species in the gas mixture is approximated by Fick's law, i.e., treating the species $i$ and the surrounding gas as a binary gas mixture, and the diffusion velocities for each species are calculated using equation 2. It should be noted that Fick's law 
is a convenient approximation of the multicomponent diffusion equations obtained from the kinetic theory of gases, and the error introduced by the approximation may become significant if the pressure gradients in the flow field are very large or molecular weights of the species are very different (ref. 12).

\section{Chemistry and Turbulence Models}

In the present study, an 8-species, 14-step chemistry model is adopted for hydrogen-air reactions. This model is a reduced $\mathrm{H}_{2}$-air reaction scheme developed from a sensitivity analysis by Hitch et al. (ref. 9) performed on a more complete model originally proposed by Westbrook (ref. 8). This reduced reaction mechanism was shown to yield good agreement with the more complete model for both the ignition delay and the reaction times over a wide range of conditions (ref. 9). It was also shown in reference 9 that the 2 -step global model developed by Rogers and Chinitz (ref. 15) predicted much shorter ignition times and longer reaction times in comparison to the 14-step model used in this report.

The closure of the governing equations is provided by the Baldwin-Lomax (B-L) (ref. 10) algebraic eddy viscosity model and constant turbulent Prandtl and Schmidt numbers $\left(P r_{t}=S c_{t}=0.9\right)$. In the formulation of the B-L eddy viscosity model, the distribution of vorticity is used to determine the length scales. The primary advantage of this model is that the boundary layer thickness which is often difficult to define in complex flows does not need to be calculated. However, it is found in the present study that in the vicinity of the injector very large vorticity is generated by the injector flow and, as a result, very large turbulent viscosities (about three orders of magnitude greater than the laminar viscosity) are calculated in this region. This large turbulent viscosity might not be physical and might affect the accuracy in predictions of the flow structure as well as the mixing between the fuel and airstream in the immediate vicinity of the injector.

Due to the highly nonlinear nature of the chemical kinetics, effects of turbulence on reaction rates can be large, especially in regions with high turbulence intensity, such as the mixing layer, recirculation zone, and in the vicinity of the fuel injector. To account for such interaction effects would require a very sophisticated turbulence reaction closure or a direct numerical simulation (DNS). Since no effective turbulent combustion model is currently available which can handle detailed finite rate chemistry and the DNS methods for complex reacting flows are still under development, the interactions between turbulence and chemistry is not considered in the present study.

\section{NUMERICAL METHOD}

\section{LU-SSOR Scheme}

Various numerical techniques have been used to solve the set of equations governing chemically reacting flows. Among these techniques, explicit schemes are generally slow in convergence when the flow involves high rates of chemical reactions and heat release or large zones of recirculation. Most implicit schemes, on the other hand, require the inversion of banded block matrices and 
become exceedingly expensive when the chemical system involves a large number of species. In the present study, the lower-upper symmetric successive overrelation (LU-SSOR) scheme of Yoon and Jameson (refs. 6 and 7 ) is adopted to solve the coupled two-dimensional Navier-Stokes and species transport equations. The LU-SSOR scheme employs an implicit Newton iteration technique to solve the finite-volume approximation of the steady-state version of the governing equations. Although the system of equations are formulated in a fully-implicit, fully-coupled manner, the LU-SSOR scheme, unlike other implicit schemes, requires only scalar diagonal inversion for the flow equations and diagonal block inversion for the species equations. As a result, the LU-SSOR scheme has the advantage of a fast convergence rate while requiring a similar operational count as that of an explicit scheme and hence is particularly attractive for reacting flows with large chemical systems. The convergence of the Newton integration method is assured by the diagonal dominance of the coefficient matrices of the LU-SSOR scheme.

In the following, for simplicity, the derivation of the LU-SSOR scheme will be presented for the Euler equations. The final formulation for the Navier-Stokes equations will be given at the end of the derivation. A prototype implicit scheme for a system of nonlinear hyperbolic equations such as the Euler equations can be formulated as

$$
\begin{aligned}
Q^{n+1}=Q^{n}-\beta \Delta t\left\{D_{x} F\left(Q^{n+1}\right)\right. & \left.+D_{y} G\left(Q^{n+1}\right)-S^{n+1}\right\} \\
& -(1-\beta) \Delta t\left\{D_{x} F\left(Q^{n}\right)+D_{y} G\left(Q^{n}\right)-S^{n}\right\}
\end{aligned}
$$

where $D_{x}$ and $D_{y}$ are difference operators that approximate $\partial / \partial x$ and $\partial / \partial y$, and $\beta$ is a positive number between 0 and 1 . Here $n$ denotes the time level. In this form, the scheme is too expensive since it calls for the solution of coupled nonlinear equations at each time step. Let the Jacobian matrices be

$$
A=\frac{\partial F}{\partial Q}, \quad B=\frac{\partial G}{\partial Q}, \text { and } \quad H=\frac{\partial S}{\partial Q}
$$

and the correction be

$$
\delta Q=Q^{n+1}-Q^{n}
$$

The scheme can be linearized by setting

$$
\begin{aligned}
& F\left(Q^{n+1}\right)=F\left(Q^{n}\right)+A \delta Q+O\left(\|\delta Q\|^{2}\right) \\
& G\left(Q^{n+1}\right)=G\left(Q^{n}\right)+B \delta Q+O\left(\|\delta Q\|^{2}\right) \\
& S\left(Q^{n+1}=S\left(Q^{n}\right)+H \delta Q+O\left(\|\delta Q\|^{2}\right)\right.
\end{aligned}
$$

and dropping terms of the second and higher order. This yields

$$
\left\{I+\beta \Delta t\left(D_{x} A+D_{y} B-H\right)\right\} \delta Q+\Delta t R=0
$$


where $R$ is the residual,

$$
R=D_{x} F\left(Q^{n}\right)+D_{y} G\left(Q^{n}\right)-S^{n}
$$

If $\beta=1 / 2$, the scheme remains second-order accurate in time, while for other values of $\beta$, the time accuracy drops to first order.

The unfactored implicit scheme (Eq. (8)) produces a large block banded matrix, which is very costly to invert and requires huge storage. Most researchers have adopted the ADI scheme which replaces the operator of equation ( 8 ) by the product of two one-dimensional operators

$$
L x L y \delta Q=-\Delta t R
$$

This scheme requires relatively expensive tridiagonal or pentadiagonal block matrix inversions. Linear stability analysis has shown that ADI schemes are unconditionally stable for two-dimensional flows. In the three-dimensional case, however, the ADI scheme is unconditionally unstable. Although artificial dissipation has some stabilizing effect, a large amount of dissipation can impair the accuracy.

Jameson and Turkel (ref. 16) proposed the idea of a lower-upper (LU) factored implicit scheme that is unconditionally stable in any number of space dimensions. There are many different ways to formulate an LU scheme (refs. 6 and 7), and here we begin with the following form of equation (8)

$$
\left\{I+\beta \Delta t\left(D_{x}^{-} A^{+}+D_{x}^{+} A^{-}+D_{y}^{-} B^{+}+D_{y}^{+} B^{-}-H\right)\right\} \delta Q=-\Delta t R
$$

where $D_{x}^{-}$and $D_{y}^{-}$are backward-difference operators and $D_{x}^{+}$and $D_{y}^{+}$are forward-difference operators. Here, two-point operators are used for steady flow calculations. $A^{+}, A^{-}, B^{+}$, and $B^{-}$are constructed so that the eigenvalues of "+" matrices are nonnegative and those of "-" matrices are nonpositive. The development of these matrices is extremely important for the success of LU-type schemes. One possibility, which yielded good results in the past (refs. 6 and 7 ) and is used in this work, is

$$
\begin{array}{ll}
A^{+}=\frac{1}{2}\left(A+\nu_{A} I\right), & A^{-}=\frac{1}{2}\left(A-\nu_{A} I\right) \\
B^{+}=\frac{1}{2}\left(B+\nu_{B} I\right), & B^{-}=\frac{1}{2}\left(B-\nu_{B} I\right)
\end{array}
$$

where

$$
v_{A} \geq \max \left(\left|\lambda_{A}\right|\right), \quad v_{B} \geq \max \left(\left|\lambda_{B}\right|\right)
$$

Here, $\lambda_{A}$ and $\lambda_{B}$ represent eigenvalues of Jacobian matrices. After manipulation (the purpose of it will become apparent later), equation (10) can be written as 


$$
\begin{array}{r}
\left\{\left[I+\beta \Delta t\left(A^{+}-A^{-}\right)+\left(B^{+}-B^{-}\right)\right]+\beta \Delta t\left[D_{x}^{-} A^{+}+D_{x}^{+} A^{-}+D_{y}^{-} B^{+}+D_{y}^{+} B^{-}\right.\right. \\
\left.\left.-\left(A^{+}-A^{-}\right)-\left(B^{+}-B^{-}\right)-H\right]\right\} \delta Q=-\Delta t R
\end{array}
$$

If we take "+" and "-" matrices as given in equation (11), then,

$$
\begin{aligned}
& A^{+}-A^{-}=v_{A} I \\
& B^{+}-B^{-}=v_{B} I
\end{aligned}
$$

Equation (13) can then be factorized to the following form

$$
\begin{aligned}
\left\{\left[I+\beta \Delta t\left(A^{+}-A^{-}\right)+\right.\right. & \left.\left.\left(B^{+}-B^{-}\right)\right]+\left[\beta \Delta t\left(D_{x}^{-} A^{+}+D_{y}^{-} B^{+}-A^{+}-B^{+}-H\right)\right]\right\} \\
& {\left[1+\beta \Delta t\left(\nu_{A}+\nu_{B}\right)\right]^{-1} I\left\{\left[I+\beta \Delta t\left(A^{+}-A^{-}\right)+\left(B^{+}-B^{-}\right)\right]\right.} \\
& +\left[\beta \Delta t\left(D_{x}^{+} A^{-}+D_{y}^{+} B^{-}+A^{-}+B^{-}\right]\right\} \delta Q=-\Delta t R
\end{aligned}
$$

It should be mentioned that the factorization of equation (14) is not unique, and among various forms we have tried equation (14) gives the fastest convergence to steady-state solutions. If $\beta=1$, the scheme reduces to a Newton iteration in the limit $\Delta t \rightarrow \infty$ :

$$
\begin{aligned}
\left(D_{x}^{-} A^{+}+D_{y}^{-} B^{+}-A^{-}-B^{-}-H\right)\left[\left(\nu_{A}\right.\right. & \left.\left.+\nu_{B}\right) I\right]^{-1} \\
& \left(D_{x}^{+} A^{-}+D_{y}^{+} B^{-}+A^{+}+B^{+}\right) \delta Q=-R
\end{aligned}
$$

It can be written as

$$
\begin{aligned}
\left(D_{x}^{-} A^{+}+D_{y}^{-} B^{+}-A^{-}-B^{-}-H\right)\left(D_{x}^{+} A^{-}+D_{y}^{+} B^{-}\right. & \left.+A^{+}+B^{+}\right) \delta Q \\
& =-\left(\nu_{A}+\nu_{B}\right)\left(D_{x} F+D_{y} G-S\right)
\end{aligned}
$$

This equation can be further reduced to

$$
\begin{aligned}
{\left[\left(\nu_{A}+v_{B}\right) I-A_{i-1, j}^{+}-B_{i, j-1}^{+}-H\right]\left[\left(\nu_{A}\right.\right.} & \left.\left.+v_{B}\right) I+A_{i+1, j}^{-}+B_{j, j+1}^{-}\right] \delta Q \\
& =-\left(v_{A}+v_{B}\right)\left(D_{x} F+D_{y} G-S\right)
\end{aligned}
$$

For the Navier-Stokes equations, $F$ and $G$ on the right-hand side of equation (17) are replaced by $F-F_{V}$ and $G-G_{V}$. That is, 


$$
\begin{array}{r}
{\left[\left(v_{A}+v_{B}\right) I-A_{i-1, j}^{+}-B_{i, j-1}^{+}-H\right]\left[\left(v_{A}+v_{B}\right) I+A_{j+1, j}^{-}+B_{i, j+1}^{-}\right] \delta Q} \\
=-\left(v_{A}+v_{B}\right)\left[D_{x}\left(F-F_{v}\right)+D_{y}\left(G-G_{v}\right)-S\right]
\end{array}
$$

Flux and Source Jacobians

In the development of flux Jacobians $A$ and $B$, evaluation of derivatives of pressure with respect to other independent variables is required. The exact formulation of these derivatives is quite complicated for flows involving real gases with nonequilibrium chemistry (ref. 17). An alternative which yields much simpler formulation is to use the concept of "equivalent Gamma." Let

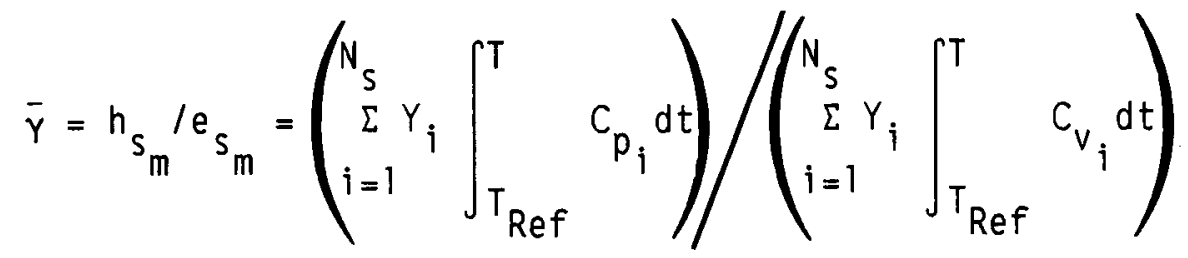

Here $\bar{\gamma}$ denotes the equivalent $\gamma$, and $h_{s_{m}}$ and $e_{s_{m}}$ are the sensible enthalpy and internal energy (excluding the kinetic energy and heat of formation) for the gas mixture. With the help of equation (19), the relationship for pressure can be written as

$$
\begin{aligned}
p & =(\bar{\gamma}-1) \rho e_{s_{m}} \\
& =(\bar{\gamma}-1)\left[\rho e-\rho h_{f_{m}}^{0}-\frac{\rho}{2}\left(u^{2}+v^{2}\right)\right]
\end{aligned}
$$

where $h_{f_{m}}^{0}$ is the heat of formation of the gas mixture,

$$
h_{f_{m}}^{O}=\sum_{i=1}^{N_{s}} Y_{i} h_{f}^{O}
$$

Since only $N_{S}-1$ species equations are actually solved, equation (20) needs to be rearranged to the following form

$$
\begin{aligned}
& P=(\bar{y}-1)\left[\rho e-\sum_{i=1}^{N_{s}-1} \rho Y_{i} h_{f_{i}}^{0}-\left(\rho-\sum_{i=1}^{N_{s}-1} \rho Y_{i}\right) h_{f_{N_{s}}}^{0}-\frac{\rho}{2}\left(u^{2}+v^{2}\right)\right] \\
& =(\bar{y}-1)\left[\rho e-\rho h_{f_{N_{S}}}^{0}-\sum_{i=1}^{N_{s}-1} \rho Y_{i} \Delta h_{f_{i}}^{0}-\frac{\rho}{2}\left(u^{2}+v^{2}\right)\right]
\end{aligned}
$$


where

\section{OF POOR QUALITY}

$$
\Delta h_{f_{i}}^{0}=h_{f_{i}}^{0}-h_{f_{N_{s}}}^{0}
$$

For real gases it is obvious that $\bar{\gamma}=\bar{\gamma}\left(e, p, \gamma_{i}\right)$. However, as discussed by Golella and Glaz (ref. 18), this function of $\gamma$ varies very slowly with the change of other thermodynamic and flow properties, and hence we have treated $\bar{\gamma}$ as a local constant in the evaluation of pressure derivatives. This approximation will not affect the accuracy of the converged steady-state solution. The final form of the Jacobian $A$ is given as

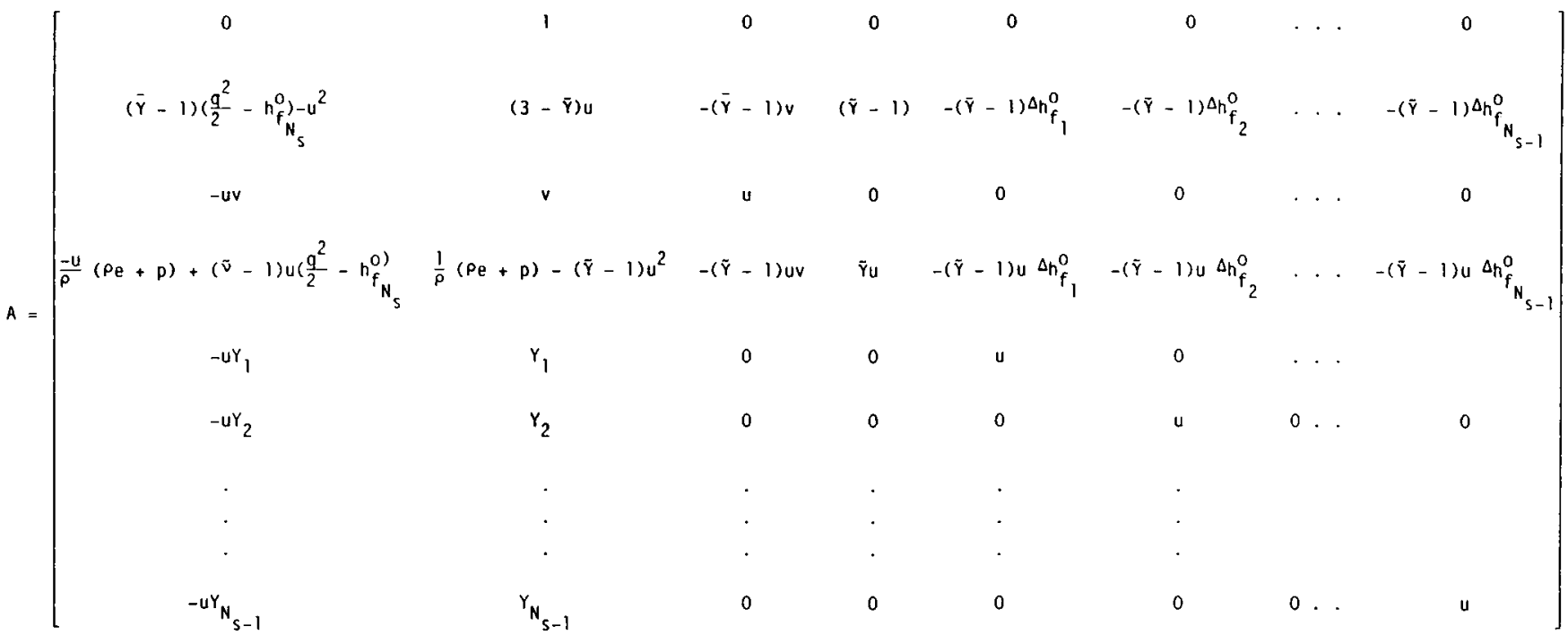

(22)

where $q^{2}=u^{2}+v^{2}$. The form of $B$ is similar to that of $A$.

Equation (18) can be rewritten in the following form,

$$
\begin{aligned}
{\left[D{ }^{A} G_{i, j}-A_{i-1, j}^{+}-B_{i, j-1}^{+}\right] } & {\left[\left(\nu_{A}+v_{B}\right) I+A_{i+1, j}^{-}+B_{i, j+1}^{-}\right] \delta Q } \\
= & -\left(v_{A}+v_{B}\right)\left[D_{x}\left(F-F_{v}\right)+D_{y}\left(G-G_{v}\right)-S\right]
\end{aligned}
$$

where $D I A G j, j=\left[\left(\nu_{A}+v_{B}\right) I-H\right]_{j}, j$ are the $\left(N_{S}+3\right) \times\left(N_{S}+3\right)$ blocks in the diagonal of the matrix operator. These diagonal blocks are of the following form, 


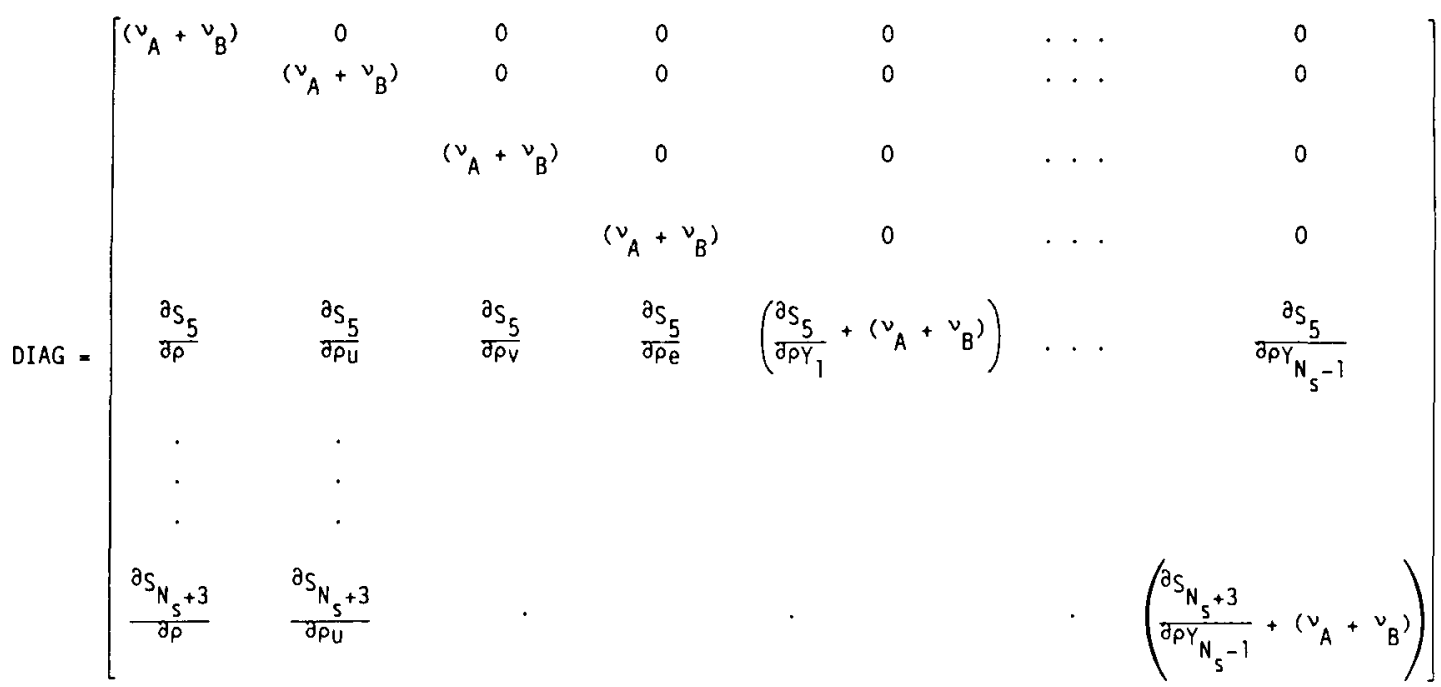

In equation (24), $\partial S_{j} / \partial \rho=0$ (since $\rho y j$ are solved as independent variables), and $\partial S_{j} / \partial(\rho u)$ and $\partial S_{j} / \partial(\rho v)$ are taken as zero since they are usually small, although they can be evaluated via chain-rule, e.g.,

$$
\frac{\partial S_{i}}{\partial \rho u}=\left(\frac{\partial S_{i}}{\partial T}\right)_{\rho Y_{i}}\left(\frac{\partial T}{\partial \bar{e}}\right)_{\rho, \rho Y_{j}}\left(\frac{\partial \dot{e}}{\partial \rho u}\right)_{\rho, \rho V, \rho e, \rho Y_{j}}
$$

where $T$ denotes temperature, $\hat{e}=e-\frac{u^{2}+v^{2}}{2}=h_{f_{m}}^{0}+\int_{T_{\operatorname{Ref}}}^{T} C_{v_{m}} d T$, and $C_{v_{m}}=\sum_{i=1}^{N_{S}} Y_{i} C_{v_{j}}$. The terms of $\partial S_{i} / \partial(\rho e)$ in equation (24) are evaluated using a chain-rule formula similar to equation (25). The $\partial S_{j} / \partial\left(\rho Y_{j}\right)$ terms can be easily calculated from the law of mass action (ref. 12).

It is interesting to note that for nonreacting flows $(\mathrm{S}=\mathrm{O}$ and $\mathrm{H}=\mathrm{O}$ in eq. (23)) the present numerical method eliminates the need for banded block matrix inversions without using the diagonalization procedure. In fact, with forward (for the first operator in eq. (23)) and backward (for the second operator) sweeps in the diagonal directions (indices $i$ and $j$ increasing or decreasing simultaneously), only scalar diagonal inversions are needed to solve equation (23) for nonreacting flow problems. For reacting flows, due to the presence of the chemical source Jacobian $H$, the first operator on the lefthand side of equation (23) now requires block diagonal inversions. However, since in the present formulation the flow equations (continuity, momentum, and energy equations) have no source terms, the first four rows of the diagonal block (DIAG) of this operator have nonzero terms only in the diagonal. As a result, the first operator of equation (23) can be inverted in essentially two separate steps: the scalar diagonal inversion for the flow equations and the block diagonal inversion for the species equations. 


\section{Boundary and Initial Conditions}

For the problems to be considered here the inflows are always supersonic, so the upstream boundary conditions are provided by specifying the velocities, static pressure and temperature, and species mass fractions. For the supersonic outflow the dependent variables are extrapolated from the interior. At the outflow plane, for grid points inside the wall boundary layers where the flow is subsonic, however, static pressure is specified (equal to the inlet pressure), and density is calculated using the ideal gas law. Along the solid wall, no-slip boundary conditions are specified. The wall is assumed to be adiabatic. The normal derivatives of pressure and species concentrations are also assumed to be zero. The injector is assumed choked (due to the high injection pressure), and hence at the injection slot all the dependent variables are fixed at their initial values. Along a plane of symmetry, the normal derivatives of all the dependent variables are zero, except for the $v$ velocity, where $v=0$ is specified.

The governing equations are intialized by setting all the dependent variables throughout the domain to the inflow conditions.

\section{RESULTS AND DISCUSSION}

One of the main purposes of this paper is to study the mixing and chemical reaction of the transversely injected hydrogen in a supersonic airstream. This flow configuration is of particular interest to the design of scramjet engines because the transverse injection increases the fuel residence time in the combustor and the flow recirculation, resulted from the injection, can help to stabilize the flame. Since the present work is the first application of the LU-SSOR scheme to combustion problems, the ability of the method to compute complex reacting flows is assessed by first computing two simpler flows where either experimental data or previous numerical results are available for comparison. These two flows are: (1) the transverse injection of helium into a supersonic airstream, and (2) the combustion of premixed $\mathrm{H}_{2}$-air supersonic flow in a ramped duct.

\section{Code Assessment}

An experiment was conducted by G.0. Kraemer and R.C. Rogers to study details of the flow field near a slot sonically injecting helium transversely into a ducted supersonic airstream. Results of this experiment were reported by Weidner and Drummond (ref. 19) along with their numerical predictions. The experimental apparatus is shown in figure 1. The conditions of the inflow air at the duct entrance are $M=2.9, T=108 \mathrm{~K}$, and $p=0.0663 \mathrm{MPa}$. The cond $\mathrm{i}-$ tions of helium at the injection slot are $M=1.0, T=217 \mathrm{~K}$, and $\mathrm{p}=1.24 \mathrm{MPa}$.

Results are given in figures 2 and 3 . Figure $2(a)$ shows the static pressure contours. The helium jet partially blocks the cross flow resulting in a bow shock and strong pressure gradients around the injector. The jet accelerates immediately after injection because of the high injector pressure and 
then forms several very strong jet shocks further downstream from the injection slot. Figure $2(b)$ presents a comparison of the calculated and experimental results for lower wall pressures upstream and downstream of the injection slot which is located at $x=0$. The calculations slightly overestimate the peak pressure as well as the distance upstream from the slot injector where the pressure begins to rise. The pressure levels immediately downstream of the injector are also somewhat overpredicted. The cross-stream static pressures and helium mass fractions at $3.81 \mathrm{~cm}$ downstream of the injector slot are shown in figure 3 . The peak pressure caused by the bow shock and the jet shocks is predicted to occur slightly closer to the lower wall than the experiment indicates. Overall, the agreement between the calculated results and the data are quite good. Considering the very complex flowfield associated with the transverse injection, the performance of the present numerical method is very encouraging.

Next case considered for code validation is the combustion of the premixed hydrogen-air supersonic flow in a ramped duct. The same flows have been computed by a number of CFD research groups (refs. 3, 4, and 20), and in the following results of the present method are compared with those of previous studies.

The geometry and inflow conditions of the two test cases $\left(T_{\infty}=900 \mathrm{~K}\right.$ and $1200 \mathrm{~K}$ ) are illustrated in figure 4 . The inflow is at temperatures below the ignition threshold. The viscous layer along the walls and the shock wave induced by the ramp increase the temperature to a value where significant reactions occur. The pressure contours for the case of $T_{0}=1200 \mathrm{~K}$ are shown in figure $5(2)$. The leading edge shocks and the ramp shock are clearly seen. Also observed is the continual increase of pressure behind the ramp shock due to heat released during combustion. Comparisons of pressure and temperature between the present calculations and the results of references 4 and 20 are given in figures $5(b)$ and $(c)$. The results are obtained along the y-station located approximately $0.13 \mathrm{~cm}$ from the lower wall. It can be seen that the agreement is reasonably good. The present calculation shows a higher level of temperature behind the ramp shock than indicated by the result of reference 20 . This is attributed to the different chemical models used in the two studies. As discussed earlier, the reaction time of the 14-step $\mathrm{H}_{2}$-air chemistry model used in this study is shorter than the 2-step global model used in reference 20, and, as a result, the present model predicts more complete chemical reactions and greater temperature rise behind the shock. Both the present results and the results of reference 20 show some oscillations in pressure immediately behind the ramp shock. This is caused by the differencing method, i.e., central differencing, used in both of the two studies. The TVD scheme used in reference 4 ( $f$ ig. $5(\mathrm{c})$ ) is seen to give a smoother pressure jump across the shock. The distributions of species mass fractions are compared next in figure 6 . The present results show larger $\mathrm{H}_{2} \mathrm{O}$ and smaller $\mathrm{OH}$ concentrations behind the shock than those of references 4 and 20 , indicating faster and more complete reactions predicted by the present chemistry model. Again, this phenomenon is attributed to the difference in chemistry models used in these studies. The comparison illustrated in figures $2,3,5$, and 6 indicates that the present results agree reasonably well with experimental data or previous numerical calculations for flows considered here, and the LU-SSOR scheme has the ability to simulate complex flows found in scramjet engines. 


\section{Mixing and Combustion of $\mathrm{H}_{2}$ Jet in a Supersonic Airstream}

To further demonstrate the capability of the present code, the mixing and combustion of a sonic transverse hydrogen jet injected from a slot into a Mach 4 airstream in a two-dimensional duct is computed. The duct geometry, inflow conditions, and the main features of the flow structure are illustrated in figure 7. The inflow conditions produce a global equivalence ratio of 0.71 (hydrogen/air mass, ratio $=0.02$ ). The convergence histories of the numerical calculations for this case and the other two cases discussed earlier are shown in figure 8. Also shown in this figure are the grid density and CPU time (on CRAY-XMP computer) for each case. Considering the fact that relatively complicated chemistry and thermodynamic and transport property models are used in these calculations, the convergence efficiency shown in figure 8 is certainly encouraging.

The velocity vectors around the injector slot are illustrated in figure 9. A large recirculation zone upstream of the injector can be clearly identified. The flow separation is caused by the adverse pressure gradient produced by the hydrogen jet and the shock-boundary layer interactions. There is also a smaller region of separation downstream of the injector caused by the blockage of flow by the hydrogen jet. In scramjet engines, the two recirculation regions provide longer fuel residence times, better mixing of fuel, air, and hot combustion gas, resulting in better flame holding capabilities for the combustor. The static pressure contours are shown in figure 10 . Both this figure and the schematic flowfield shown in figure 7 indicate that the transverse hydrogen jet partially blocks the axial flow and generates a strong bow shock just ahead of the injector. The hydrogen jet accelerates to supersonic speed after injection and then decelerates by jet shocks not too far from the injector. The incidence and reflection (by the up-running bow shock from the bottom half of the duct) of the bow shock to and from the symmetric plane of the duct can also be seen in figure 10. The contour maps of the mass fractions of two major combustion products, i.e., $\mathrm{H}_{2} \mathrm{O}$ and $\mathrm{OH}$, are presented in figure 11. The large mass fractions of $\mathrm{H}_{2} \mathrm{O}$ and $\mathrm{OH}$ found in the recirculation zone in front of the injector indicate that transverse injection of fuel provides good flameholding capability for the simulated scramjet combustor. Figure 11 also indicates that the injected fuel penetrates well into the mainstream, and good mixing and chemical reaction occur in the combustor.

\section{CONCLUDING REMARKS}

A computer code for analyzing two-dimensional chemically reacting flow fields has been developed. This code employs the LU-SSOR finite volume scheme which solves the Navier-Stokes equations and species transport equations in a fully implicit and coupled manner. The validity of this code is demonstrated by comparisons of the present calculations with experimental data and previous numerical results. The code has been used to simulate the flowfield in a scramjet combustor in which the hydrogen is transversely injected into a supersonic airstream. The present results show that the new code can handle the complex flowfield associated with the fuel injection, mixing, and chemical reaction in a scramjet combustor. The new code has also been shown to be very efficient and robust for the flows considered in the present study. 
Interactions between the turbulence and chemical reactions are not accounted for in the present study. The interaction effects are important in most ramjet/scramjet combustor flows, especially for simulating ignition, flameholding stability, and heat release rate, and should be considered in the future code improvement effort.

\section{REFERENCES}

1. Bussing, T.R.A., and Murman, E.M., "A Finite Volume for the Calculation of Compressible Chemically Reacting Flows," AIAA Paper 85-0331, Jan. 1986.

2. Eklund, D.R., Hassan, H.A., and Drummond, J.P., "The Efficient Calculation of Chemically Reacting Flow," AIAA Paper 86-0563, Jan. 1986.

3. Uenishi, K., Rogers, R.C., and Northam, G.B., "Three Dimensional Computations of Transverse Hydrogen Jet Combustion in a Supersonic Airstream," AIAA Paper 87-0089, Jan. 1987.

4. Yee, H.C. and Shinn, J.L., "Semi-Implicit and Fully Implicit ShockCapturing Methods for Hyperbolic Conservation Laws with Stiff Source Terms, " 8th Computational Fluid Dynamics Conference, AIAA, New York, 1987, pp. 159-176. (NASA TM-89415).

5. Beam, R.M., and Warming, R.F., "An Implicit Finite-Difference Algorithm for Hyerbolic Systems in Conservation-Law Form," Journal of Computational Physics, Vol. 22, No. 1, Sept. 1976, pp. 87-110.

6. Yoon, S., and James, A., "An LU-SSOR Scheme for the Euler and NavierStokes Equations," AIAA Paper 0600, Jan. 1987.

7. Jameson, A., and Yoon, S., "Lower-Upper Implicit Schemes with Multiple Grids for the Euler Equations," AIAA Journal, Vol. 25, No. 7, July 1987, pp. 929-935.

8. Westbrook, C.K., "Hydrogen Oxidation Kinetics in Gaseous Detonations," Combustion Science and Technology, Vol. 29, No. 1-2, 1982, pp. 67-81.

9. Hitch, B.D., Laxter, W.R., Senser, D.W., and Sojka, P.E., "On the Selection of $\mathrm{H}_{2} / \mathrm{Air} \mathrm{Kinetic}$ Mechanisms for Use in Supersonic Combustor Modeling," presented at the Spring 1986 Technical Meeting of the Central States Section of the Combustion Institute, Cleveland, OH, May 1986.

10. Baldwin, B. and Lomax, H., "Thin Layer Approximation and Algebraic Model for Separated Turbulent Flows," AIAA Paper 78-257, Jan. 1978.

11. Shuen, J.S., "Effects of Droplet Interactions on Droplet Transport at Intermediate Reynolds Numbers," AIAA Paper 87-0137, Jan. 1987.

12. Williams, F.A., Combustion Theory, 2nd ed., Benjamin/Cummings Publishing Company, Men lo Park, CA, 1985, pp, 631-645.

13. McBride, B.J., private communication, NASA Lewis Research Center, Cleveland, OH, Apr. 1987. 
14. Reid, R.C., Prausnitz, J.M., and Sherwood, T.K., The Properties of Gases and Liquids, 3rd Ed., McGraw-Hill Publishing Company, NY, 1977.

15. Rogers, R.C., and Chinitz, W., "Using a Global Hydrogen-Air Combustion Model in Turbulence Reacting Flow Calculations, "AIAA Journal, Vol. 21 , No. 4, Apr. 1983, pp. 586-592.

16. Jameson, A. and Turkel, E., "Implicit Schemes and LU Decompositions," Mathematics of Computation, Vol. 37, No. 156, 1981, pp. 385-397.

17. Schuen, J.S., Liou, M.S., and Van Leer, B., "A Detailed Analysis of Inviscid Flux Splitting Algorithms for Real Gases with Equilibrium or FiniteRate Chemistry," paper submitted to the 11 th International Conference on Numerical Methods in Fluid Dynamics, June 1988.

18. Colella, P., and Glaz, H.M., "Efficient Solution Algorithms for the Riemann Problem for Real Gases," Journal of Computational Physics, Vol. 59, No. 2, June 1985, pp. 264-289.

19. Weidner, E.H., and Drummond, J.P., "Numerical Study of Staged Fuel Injection for Supersonic Combustion," AIAA Journal, Vol. 20, No. 10, Oct. 1982, pp. 1426-1431.

20. Chitsomboon, T., Kumar, A. and Tiwari, S.N., "Numerical Study of FiniteRate Supersonic Combustion Using Parabolized Equations, "AIAA Paper 87-0088, Jan. 1987.

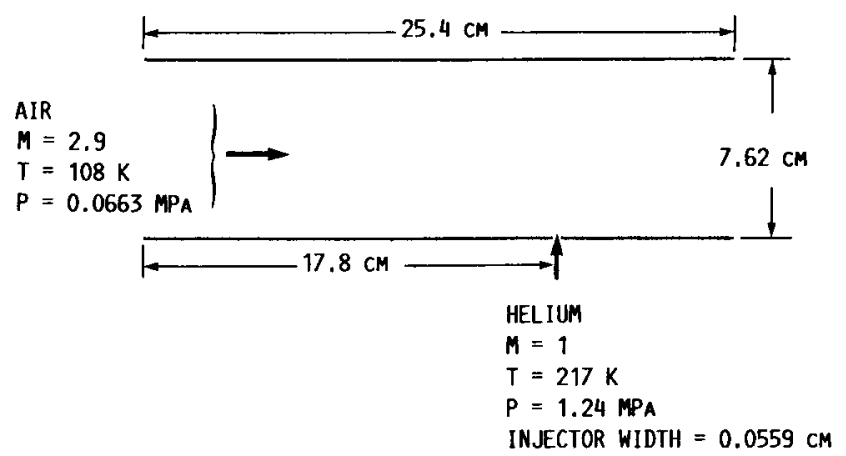

FIGURE 1. - GEOMETRY AND INFLOW CONDITIONS FOR THE MIXING OF AIR AND A TRANSVERSE $\mathrm{H}_{\mathrm{e}}$ JET.

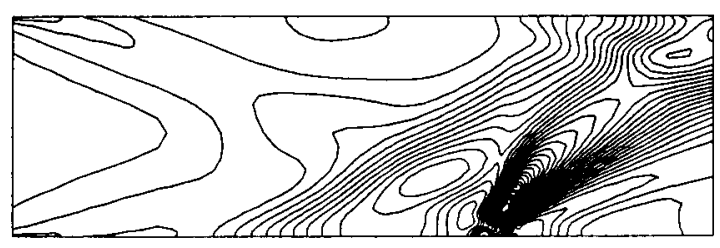

(A) STATIC PRESSURE CONTOURS.

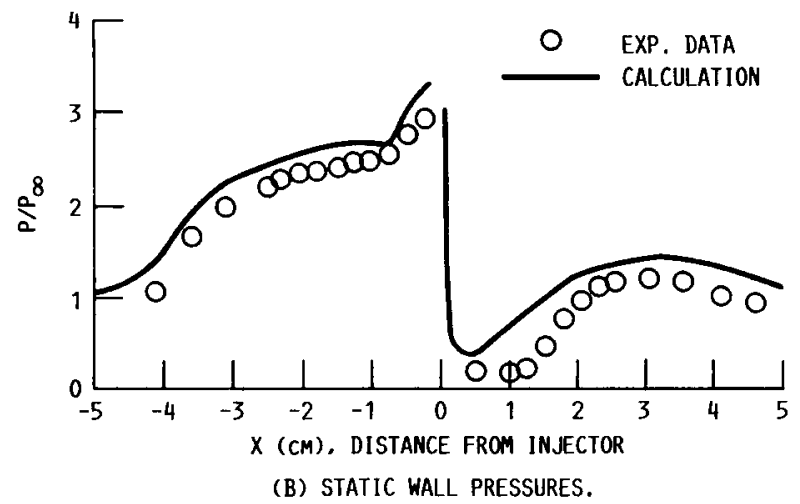

FIGURE 2. - STATIC PRESSURE CONTOURS AND STATIC WALL PRESSURES OF THE TRANSVERSE $\mathrm{H}_{\mathrm{C}}$ INJECTION CASE. 


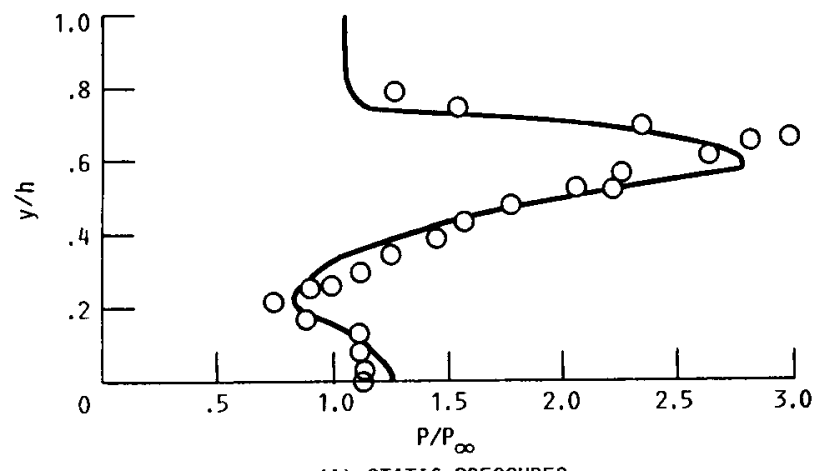

(A) STATIC PRESSURES.

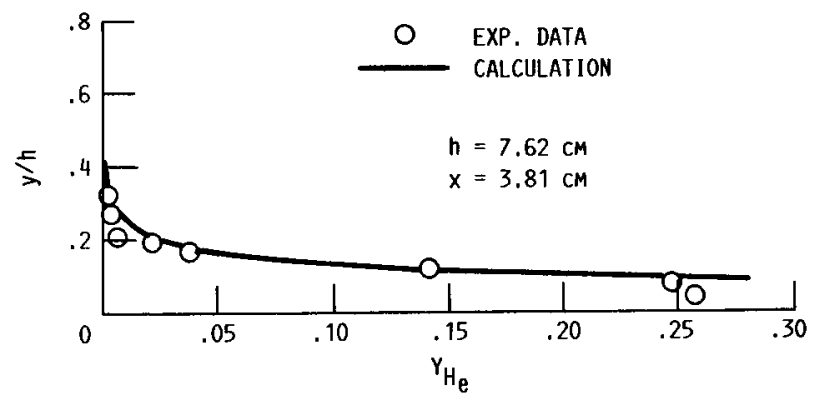

(B) HEL IUM MASS FRACTIONS.

FIGURE 3. - COMPARISON OF EXPERIMENTAL AND COMPUTED STATIC PRESSURES AND HELIUM MASS FRACTIONS.

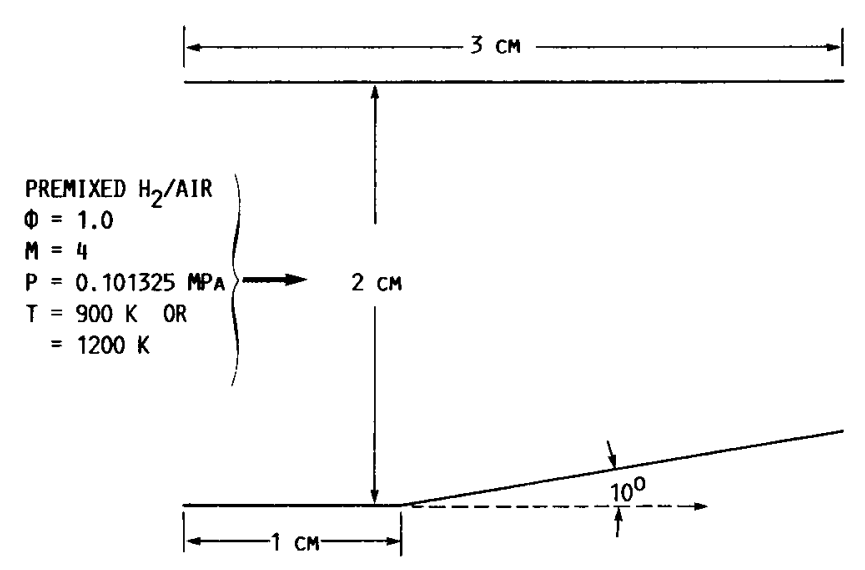

FIGURE 4. - GEOMETRY AND INFLOW CONDITIONS FOR THE PREMIXED $\mathrm{H}_{2} /$ AIR REACTING FLOWS. 


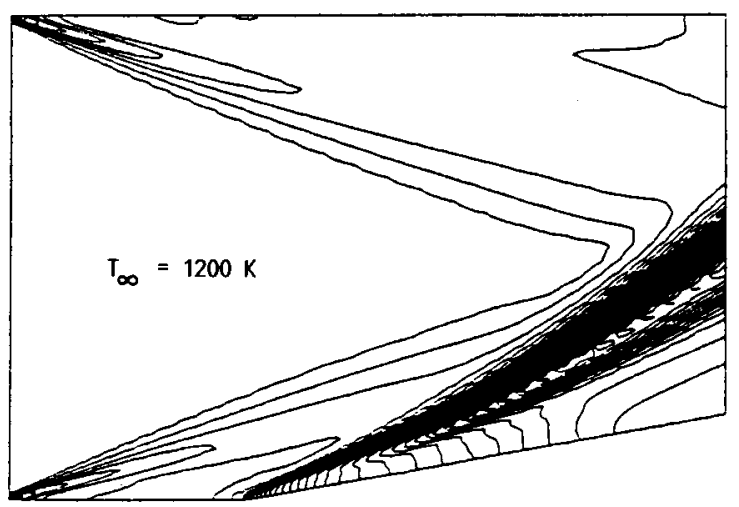

(A) STATIC PRESSURE CONTOURS.

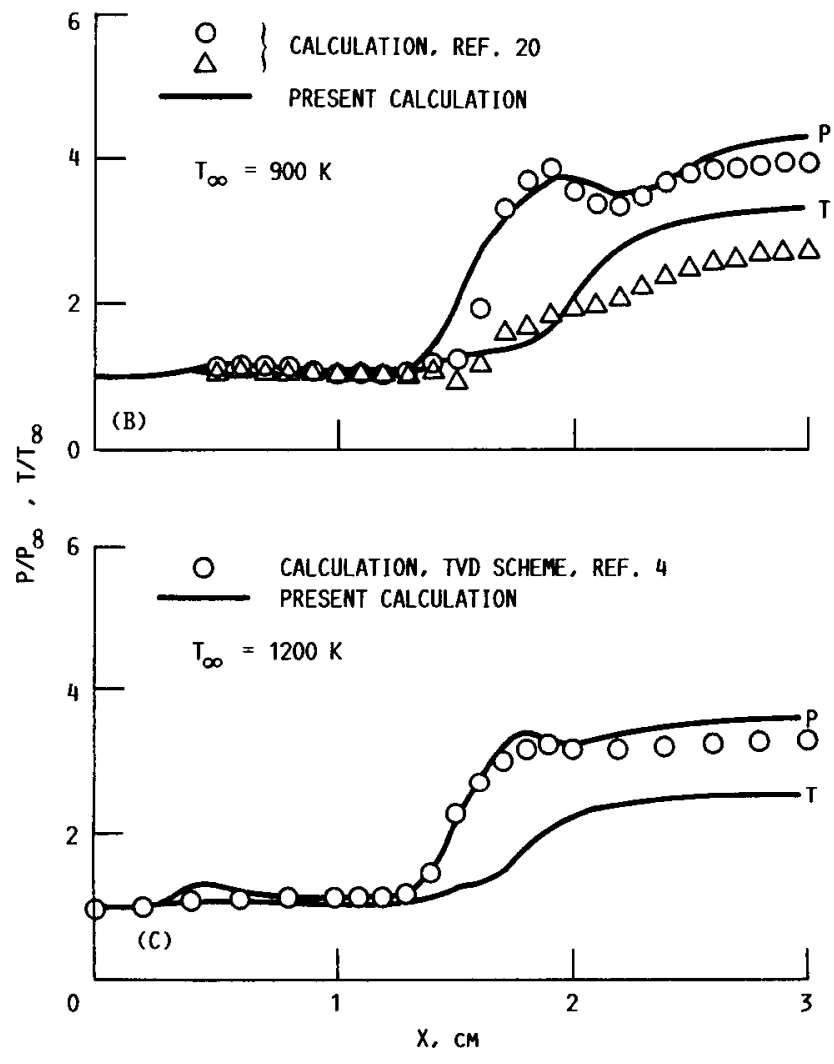

FIGURE 5. - STATIC PRESSURE CONTOURS, PRESSURE AND TEMPERATURE ALONG $y=0.13 \mathrm{~cm}$.

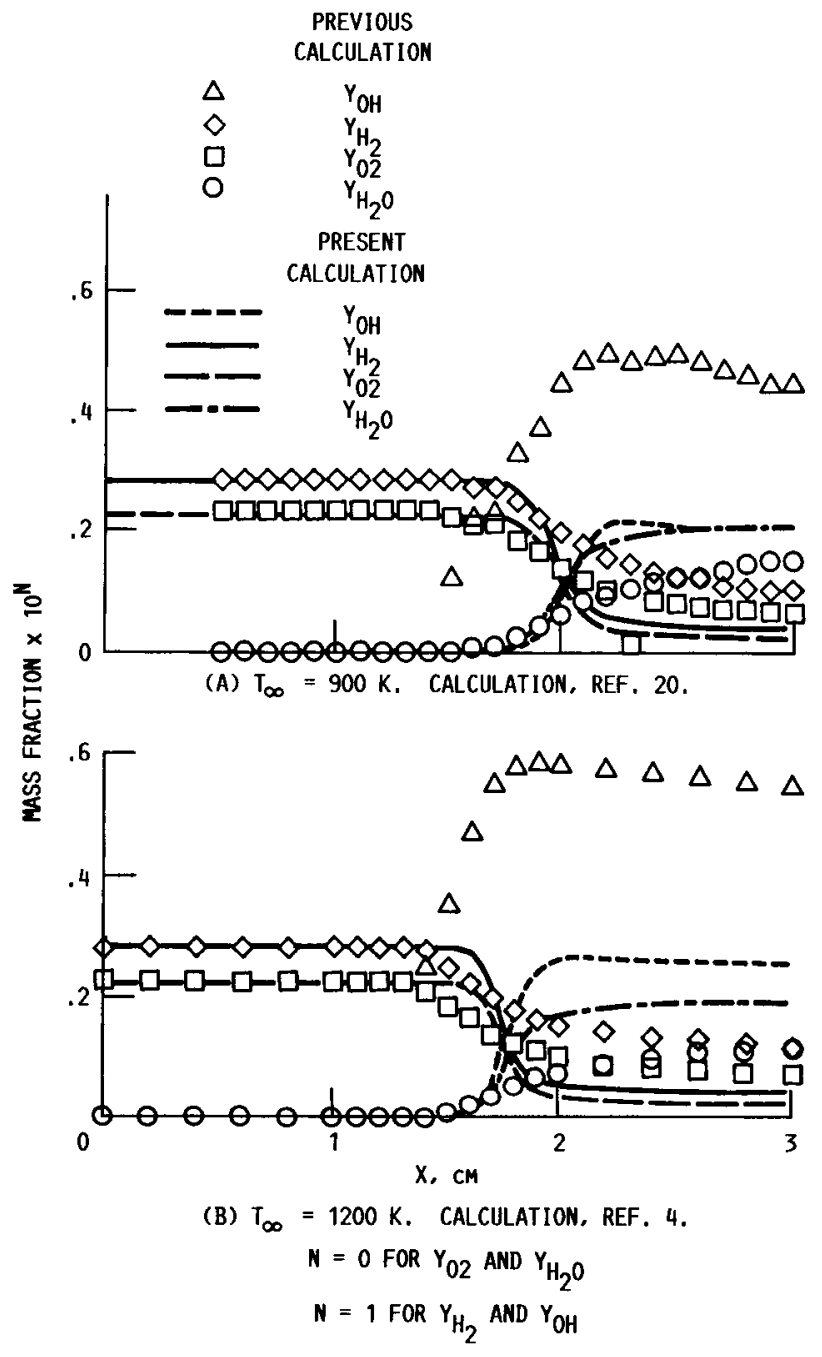

FIGURE 6. - COMPARISON OF SPECIES MASS FRACTIONS BETWEEN PRESENT AND PREVIOUS NUMERICAL PREDICTIONS. 


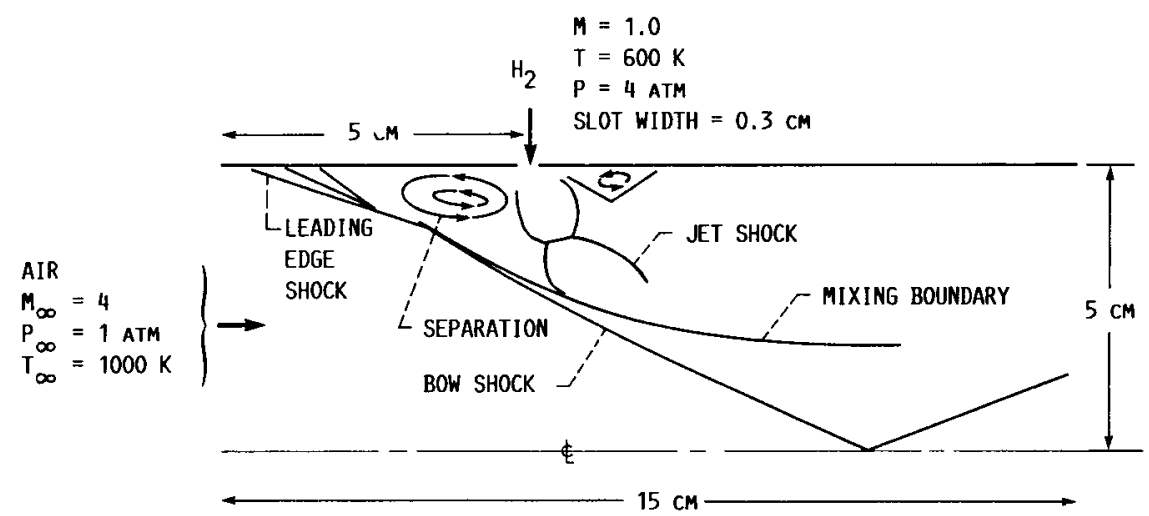

FIGURE 7. - GEOMETRY AND INFLOW CONDITIONS OF THE TRANSVERSE HYDROGEN INJECTION CASE.

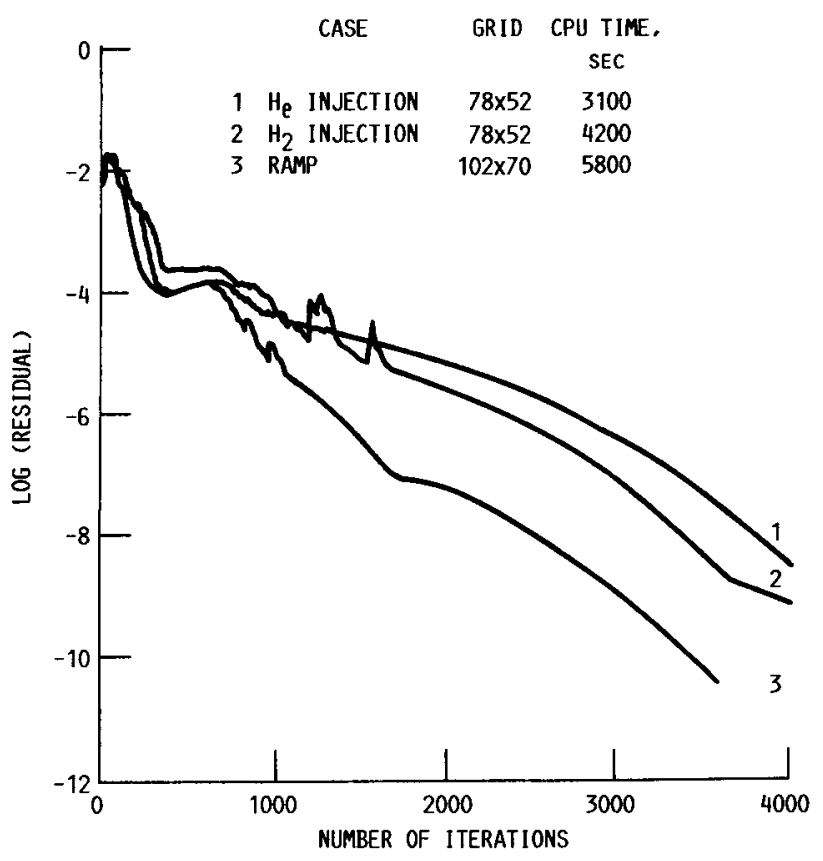

FIGURE 8. - CONVERGENCE HISTJUR OF NONDIMENSIONALIZED $\mathrm{L}_{2}$ DENSITY RESIDUAL. 


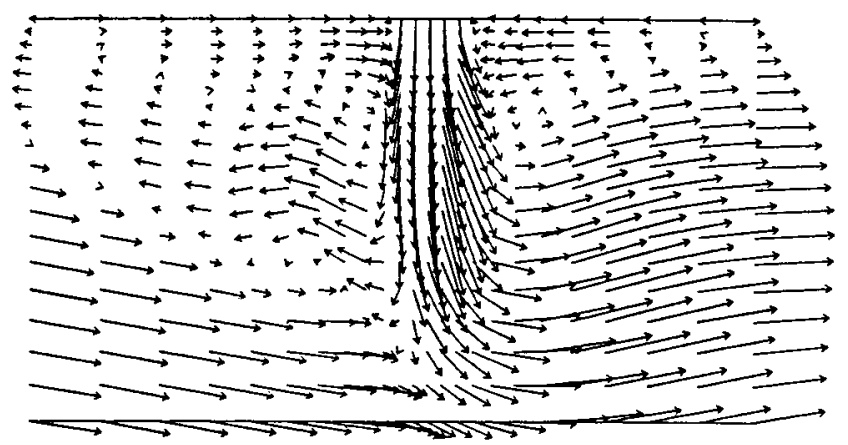

FIGURE 9. - VELOCITY VECTORS AROUND THE INJECTOR FOR THE $\mathrm{H}_{2}$-INJECTION CASE.

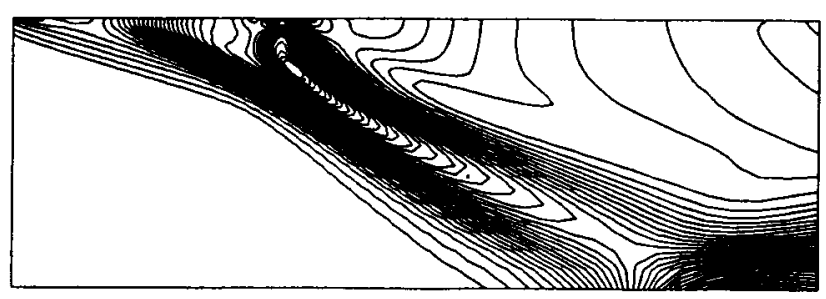

FiguRE 10. - STATIC PRESSURE CONTOURS FOR THE H $\mathrm{H}_{2}$-INJECTION CASE.

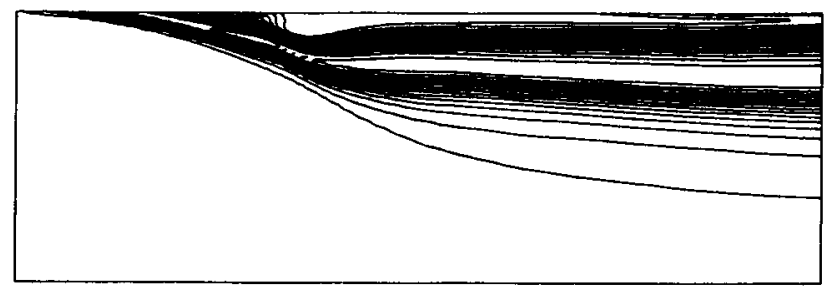

(A) $\mathrm{H}_{2} \mathrm{O}$ MASS FRACTION CONTOURS.

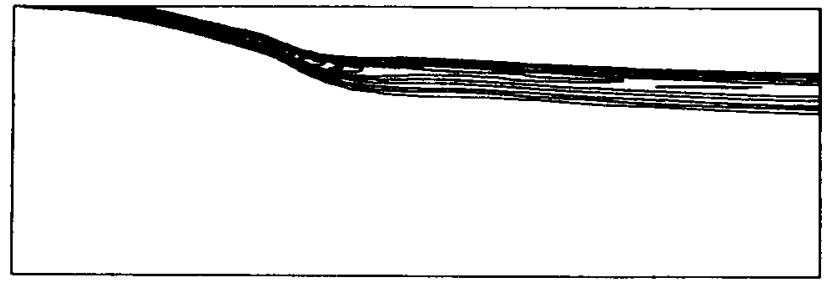

(B) OH MASS FRACTION CONTOURS.

FIGURE 11. - $\mathrm{H}_{2} \mathrm{O}$ AND OH MASS FRACTION CONTOURS FOR THE $\mathrm{H}_{2}$-INJECTION CASE. 


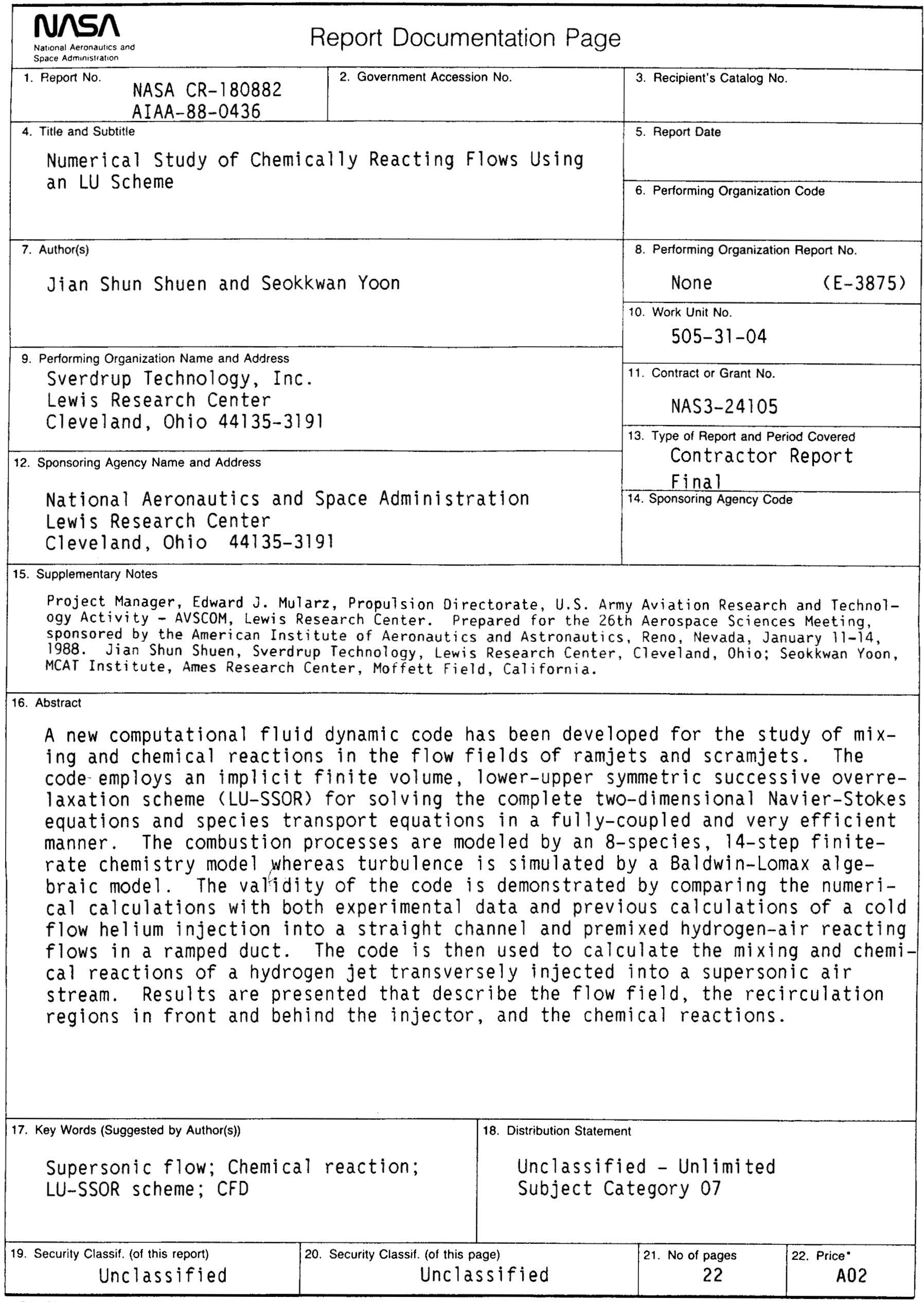

